\title{
Patchwork, Functions and Origin of Clothing for Women in Jiangnan Watery Region of China
}

\author{
Jingqiong Zhang \\ Han Chinese Costume Museum \\ School of Textiles and Clothing \\ Jiangnan University \\ Wuxi, Jiangsu 214122, China \\ E-mail: ZHANG_Jingqiong @yahoo.com \\ Tengteng Ge (Corresponding author) \\ School of Textiles and Clothing \\ Jiangnan University \\ Wuxi, Jiangsu 214122, China
}

Tel: 86- 510-8591-9591 E-mail: GE_Tengteng@yahoo.com

Yanju Cao

School of Fashion Design

Shandong University of Art \& Design

Jinan, Shandong 250014, China

E-mail: hotcatmm@163.com

Foundation item: Humanity and Social Science Funds of the Ministry of Education of P. R. China. (No.08JA760010)

\begin{abstract}
Patchworking is a kind of needlework technique in which two or more pieces of cloth are sewn together. It is commonly used for making female clothes in the countryside east of Suzhou, China. The practicalities of the patchwork are manifold: economy, easy replacement of the patches, which can be made so to hold small items and to strengthen the waist of the body. On the aesthetic side, the proportionality of the patches, the original patterns of blessing woven in is simply a delight to look at. Its history can be traced back to the traditional costumes developed throughout the Song, Ming, and Qing dynasties.
\end{abstract}

Keywords: Women's clothing, Patchwork, Functions, Origin, Jiangnan watery region, China

\section{Introduction}

Women's clothing in east Suzhou of Jiangsu province displays some remarkable district characteristics. The region locally called "Wu East", where the famous old towns Luzhi and Zhouzhuang are located, includes the Yangcheng Lake, Cheng Lake, Dianshan Lake and the Baimao area. Crisscrossed by rivers, climate here features four distinct seasons. Local inhabitants mainly engage in rice production. Therefore, the custom developed here is called "Rice Cultivation" which is intrinsically related to their way of life. Still used in modern times, the unique clothing for womenfolk in water countries is part of Rice Cultivation Culture. A complete set of clothing for women in Jiangnan watery region and which 
is wrapped around the human body can be described as following: 1. Head scarf - - head; 2. Chinese-style blouse - trunk and upper limbs; 3. Crotch-jointing Pants- - trunk and lower limbs; 4. Waist-skirt - - trunk; 5. Pleated skirt - trunk and lower limbs; 6. Bellyband - - trunk; 7. Bandage - - the lower limbs; 8. Hundred-stitch embroidery Shoes - feet.(See Fig. 1 A Complete Set of Clothing for Women in Jiangnan Watery Region).

\section{The Patchwork}

As previously introduced, the patchwork is a kind of needlework in which two or more pieces of cloth are sewn together to make a garment. People usually cut a whole piece of cloth into squares or various shapes as required, and piece these together using a sewing technique. Because the cloth are fully made used of, including the odds and ends, so the patchwork can be considered an important strategy in saving fabrics which otherwise would be wasted. The design and craft of the patchwork involved in the making of women's clothing in Jiangnan watery region are very popular, and are mainly embodied in the following clothing:

\subsection{The head scarf}

When unwrapped, the head scarf is shaped as an isosceles trapezoid and forms two triangles similar to coattail after binding, hence called "triangle headdress" in the local community. "The top edge of the head scarf is usually about 60 to $70 \mathrm{~cm}$ long while the bottom edge is 100 to $110 \mathrm{~cm}$ long and the head scarf is 25 to $28 \mathrm{~cm}$ wide. The bevel edge of the head scarf is slightly arc-shaped. There is a sword shaped ribbon or a rope with tassels approximately $10 \mathrm{~cm}$ in length at each top end for the purpose of binding the top."(Zhang, 2005, P.133).The head scarf is generally made of black cloth, called "top". The head scarf has two sides made of two small pieces of cloth in different colors, such as white and blue, which is called "angles", namely "two-color joint angles". Some angles are made of three small pieces of cloth in different colors, namely "three-color joint angle". Usually embroidery is rarely seen in two-color patchwork but common in three-color patchwork and embroidery is on the top of the patchwork. Where there are colors combination there must be an embroidered border. According to local customs, girls who are more than thirteen years old begin to wear head scarf after keeping hairs. During this period, the color of their head scarf is a little lighter and with no patchwork. When they are over fifty, the color grows darker and becomes black and dark gray and with little patchwork. However, the head scarf of local women between 30 and 40 years old display many colors and joint angles, including black top with white angles, black top with blue angles, blue top with white angles, green top with black angles and etc. These head scarf are usually made of plain cotton, homespun or burnt-out print and can be single layer or double layers. (See Fig.2 Local Women's Head Scarf \& Fig.3 Structure of the Local Head Scarf).

\subsection{The Chinese-style blouse}

The Chinese-style blouse in this region doesn't differ in styles from those of the other regions of China. The slanting front of the Chinese-style blouse is uniformly right and with more patchwork in waterside villages along the Yangtze River. Vertical and horizontal styles are considered as two forms of the patchwork. The vertical style literally means the patchwork vertically finished in the middle of sleeves. The right front and left front can be different or the same in color. While the horizontal style, literally means the patchwork horizontally finished at the waistline. Colors above and beneath the waistline are different from each other. The vertical style is popular while the horizontal style is rarely seen. Two styles can also be used in combination. Namely a vertical patchwork is made at half of the sleeves and then a horizontal patchwork is made at the waistline. The two parts exhibit different colors, locally called "Guanjiantou" (Fig.4). Owing to the fabric square limitation, the sleeve usually has only one patchwork finished and likes "Zhaoxiu" of traditional costume. Sometimes it has two patchworks finished and is divided into three pieces. Chinese-style blouse are considered as the main upper outer garments for local women. They wear it not only when working in fields and living in houses but also when shopping in town-markets. (See Fig. 4 Local Women's Blouse \& Fig.5 Structure of the Local Blouse).

\subsection{The waist-skirt}

The Waist-skirt is an apron of double layers fastened by waistband outside the Zuo-skirt. It is usually made of mixed color fabrics. No matter it is the upper or the lower layer, each layer is divided into three parts by two vertical patchworks. One color is applied to the middle part of each layer and another color is applied to its two sides. Both the two layers shape in trapezium. The upper part of the waist-skirt is called waistband which is $18 \mathrm{~cm}$ long and $5 \mathrm{~cm}$ wide. Commonly the belt-shape waistband is daintily sewed and with pieces of starched cloth padded which seems very thick, hence it is locally called "Waist-Crossed Board". Attached to the waist-skirt by buttons, the waistband acts like a wide and thick belt binding around the waist. (See Fig.6 Local Women's Waist-skirt \& Fig.7 Structure of the Local Waist-skirt).

\section{Functions}

\subsection{Practical functions}

Practical functions of the head scarf are conspicuously related to farm work. The head scarf acts like a barrette which 
keeps women's hairs from hanging down while transplanting rice seedlings. The long angles of the head scarf protect the neck from the scorching sun and, wrapped around the head, it keeps the body from the cold wind in winter. Furthermore, it safeguards women from flies' biting and keeps their hair from dirty working in the fields. Therefore, it is almost certain that the head scarf of this particular make is formed by the local mode of rice production, taking into the fact that all you need to make the joint angles of the head scarf is the odds and ends of cloth thus saved.

The functional meaning of the patchwork for the Chinese-style blouse lies, first of all, in easy removal and convenient replacement. "Three pieces of different fabrics are applied to make the patchwork on shoulders, sleeves and the main body of the Chinese-style blouse. This is because the above-mentioned parts tend to wear away easily during local women's labor, including carrying, planting and etc. The main body of the garment can be worn for a longer time after the replacement worn-out parts. Skillfully prolonging the service life of their garments, they never wear clothes with patches. So their clothes always seem to be new or nearly new (Zhang, 2002, PP.86-88)!" Good practical and aesthetic effects are achieved with relatively less cost due to the patchwork. Secondly, the patchwork is made for the purpose of saving fabrics. According to ancient record, fabric width $2 \mathrm{chi}$ and $2 \mathrm{cun}$ in ancient China is more than $50 \mathrm{~cm}$ today. (chi and cun are traditional Chinese units of length.) The width of fabric woven by local women about 9 cun to 1 chi is more than $30 \mathrm{~cm}$ today. Even the early machine-woven single width cloth is measured only 90 . Therefore, the patchwork is a result of fabric width limitation. Assuming that the clothing layout is subject to the widest fabric width of $90 \mathrm{~cm}$ which is folded then, the garment doesn't need the patchwork for the front and back. However, the patchwork for the sleeve is required. If the fabric width is not folded, the result turns out to be opposite. In case that the clothing layout is subject to the width of hand woven fabric, the garment needs the patchwork for both the front and back and sleeves. What's more, joint angles for both sides of the garment bottom are required as well. All are done reluctantly by local women.

The functional significance of the patchwork for the waist-skirt lies, first of all, in saving fabrics. The size of a waist-skirt is measured. Each side part of the outer layer is $23 \mathrm{~cm} * 6 \mathrm{~cm}$ and the middle part is $23 \mathrm{~cm} * 18 \mathrm{~cm}$. Each side part of the inner layer is $26 \mathrm{~cm} * 10 \mathrm{~cm}$ and the middle part is $26 \mathrm{~cm} * 20 \mathrm{~cm}$. Local women can't bear to make the inner layer with the whole piece of cloth for the reason that its middle part is covered by the outer layer. Among ten waist-skirts treasured by the author, all the middle parts of the inner layers are made up of small pieces of cloth thus saved. The very smallest pieces of cloth are full used of through this way. The waist-skirt is connected to the waistband with buttons and if it is worn out after many days, the waistband full of embroidery can be untied and applied to a new waist-skirt. Secondly, the waist-skirt itself can strengthen the waist of the body for local women who engage in prolonged farm work in the rice fields and have to bend down frequently. Because the waistband is daintily sewed with several layers and covered with embroidery, it is thick and hard enough to strengthen the waist of body and relieve fatigue. It can be concluded that the style of the waistband is a wonderful expression of the Rice Cultivation Culture. The waist-skirt can be used to hold small items as well. Some important things such as bills, notes and paper money can be put into the patch pocket in the position of the lower part of the waist-skirt. In that case, the outer layer of the waist-skirt has been naturally and skillfully turned into the flap of the pocket! There was an interesting story occurred during the author's purchasing: one local woman conveniently untied her waist-skirt to sell it and even forgot to take out the money from the pocket. Last but not least, the waist-skirt can keep Zuo-skirt clean by hiding it inside. Obviously, it is much easier to wash the waist-skirt than the Zuo-skirt because the waist-skirt is smaller in size.

\subsection{Psychological functions}

Patchworking technique firstly has been adopted for the purpose of saving fabrics because of fabric width limitation. Later, patchworking with fabrics in the same color was developed into patchworking with fabrics in multiple colors, and the objective need is developed into subjective seeking.

The psychological satisfaction behind the attractiveness of the women's clothing may be summed up as follows: The first is the practicality part of the attraction. The patchwork was originally made for the purpose of utility. Thus, utilitarian meanings such as saving, fabric width and replacement are implied both in lines and color blocks of the patchwork. This is one of the characteristics of folk creations and it also displays an essential difference between civilian creations and official creations. From citizens' view, utility is the most essential and original purpose, which is deliberately developed. After all, their clothing is a kind of daily necessity in labor time and daily life. As a result, it may bring about another by-product "beauty" as it satisfies practical needs. The patchwork brings us a sense of face shape and the additional embroidered border (shaped like a thin joss stick) originally designed for a further safeguard of the neckband, the wristband as well as the front bring us a sense of line shape as the saying goes "many things grow in the garden that were never sown there". Then affected by this by-product, people try to diversify the patchwork in fabrics and colors and strive for the combination of economy and decoration. This decoration beautifies daily necessities or it is a kind of beautified daily necessity itself when aesthetic significance exceeds the practical meaning.

Secondly, the beauty of the shape and proportionality of cloth that goes to the making of patchwork clothing. Principle of creation as manufacturing according to the nature of the substance and using according to the size of the materials in Jiangnan water villages reflects the virtue of thrift itself. So not only perfection, but also supreme goodness is achieved. 
In fact, the harmony of number and economy has been considered in the process of making head scarf and waist-skirt with small pieces of cloth. Originated from this principle, embroidered fabrics are pieced together at the corner of the head scarf. The waist-skirt thus pieced formed the length and width of sides and the middle close to the gold ratio. On the whole, symmetry joins are applied to two corners of the head scarf and the hem, two sleeves of the Chinese-style blouse as well as the waist-skirt, meeting the demand of the spirit and forming strong color contrast and harmonious proportion relationship. As a local proverb goes, "it is just for showing”. However, this kind of showing demonstrates not women's figure and curve but the beauty of clothing, displaying the connotation of aesthetic standard for Chinese clothes which is different from taste of the west. It is well justified by a Chinese proverb, saying that "the field has its special use and people have their own wisdom". Besides, it reflects Chinese people's intelligence and esthetic taste, which is another specialty of Chinese folk creation.

Thirdly, the creativeness in the daily life of the folk is part of the beauty we see in the clothes for women in areas along Southern Yangtze River. Local women's clothes, an important part of their house needlework, are made in off-seasons and worn in busy farming seasons. Free styles and plain design of the clothing express the original purity and clever perception, full of childlikeness. Local women can share patterns and designs from neighbor girls, and also can apply their own inspirations into their clothes. The size and color of the cloth preserved in home can also decide the style and design of the clothing. Local women can add small changes and details into the traditional styles, for example, they change the details of the patchwork as their will to combine technique with artistic creation. Actually it is a way to transmit culture. Real objects which act as the medium include head scarf, waist-skirt, embroidery, the pattern and such articles. During the transmitting process, users will not be the original creators for some recreations have been made consciously or unconsciously when duplicating. Furthermore, regional scope limitation was formed due to the inconvenient traffic in ancient times. Usually pattern of clothing in either side of a river or a mountain can be different from each other. For example, Luzhi and Shengpu are both small towns in Jiangnan watery region and they are very close to each other, however, the head scarf in Luzhi town is obviously different from that in Shengpu in many ways, such as that the angle of head scarf in Luzhi town is longer and the head scarf in either town has its special characters. Local women are honest, simple and frank and without too much strategy and shrewdness (Hu, 1984, p.433), used to gain happiness through their working hands. Therefore, the piped decoration made on local women's clothes is simple and the joint color is symmetric. It is not creation for creation itself but just for life. The way it is created displays the difference between arts and handicraft.

Fourthly, the beauty comes from the auspicious blessing bestowed in the costumes. Patchwork of different colors not only gives an eye-grabbing effect to the head scarf and waist-shirt and enriches the beauty of the person wearing them but also have a meaning of auspicious blessing. It is typically expressed by embroidery at the corner of the head scarf and waist-skirt which usually adopt patterns that look like the skin texture of carp fish or butterfly peony and so on, symbolizing some beautiful wishes. Compared with the other regions of China, women's clothes in Jiangnan watery region don't have much embroidery because cotton and flax are popular there for cheapness and durability which make them easily available to the patchwork. Another reason for more patchwork but less embroidery for local clothing is that the patchwork has achieved the aesthetic feeling and there is no need for embroidery or the effect will be ruined. Therefore, embroidery for the head scarf display only at the corner of the joint angles and usually local women stick a flower on bob to enrich their head adornments.

\section{The origin}

The history of patchworking technique can be traced back to ancient times of China. One of the ancient costumes was Shen clothing, a combination of coat and skirt. Therefore, there must be some patchwork between the coat and the skirt. It is a result of transition from the separation of coat and skirt to the robe shape. The Shen clothing has twelve pieces representing twelve months of the year (Zhou, 1984, p.50). It has now become a mystery as to how it was actually made, people generally agree however that it must have been pieced together, the origin of patchworking in Chinese costumes. "The upper outer garments excavated from HUANG Sheng's tomb of Southern Song Dynasty in Fuzhou city is made up of four parts including the collar, sleeves, the front and back. Some sleeves are connected to the main body of the garment and some are sewn with two small pieces because of fabric width limitation. Two pieces of the back parts are sewn together with an obvious back line from the top to the bottom. All these four parts of the costume are made of different fabrics." (Fujian Museum, 1977, p.4).This archaeological discovery indicates that there were patchworks of different fabrics in ancient costumes. Likewise, robes, coats and lined coats are unearthed from Mrs. Zhou's tomb of Southern Song dynasty in De'an, Jiangxi province. Fabrics are folded up twice according to the cloth length and tailored subject to the half of the hem, waist and the whole sleeve width. A piece of small triangle crotch is respectively added at the crotch position (Jiangxi Provincial Institute of Cultural Relics and Archaeology, 1990, p.4). All these solutions to the limitation of fabric width in ancient times of China are maintained by women in Jiangnan watery region, demonstrating comings and goings of the patchwork and its corresponding tailoring technique. A pleated skirt, unearthed from the tomb of couple XU Fan of Ming dynasty in Taizhou of Jiangsu Province, is actually patchwork skirt(Taizhou Museum, 1986, p.3), justified by a historical book--- Hu Bu Hu Lu which says that "the waist position of 
the skirt was interrupted by a line and the bottom was pleated, named Chengzi garment". ZHOU Xi-bao thought it was just the "big pleat" mentioned in the book Ming Gong History (Zhou, 1984, P.433). "Big Pleat" appears between the coat and the skirt, whose origin is the same as that of Shen clothing. As for the robe and lined coat in Qing dynasty, actually, center seam is always applied to front and back and the seam is always applied to sleeves owing to fabric width limitation. Besides the patchwork and embroidery for the collar, front and hem, there is also "Langan" for sleeves---an exceedingly skillful and luxurious patchwork applied to the sleeve and cuff. Obviously, the practicality of the patchwork has been changed to aesthetic considerations and the positive attitude has been changed to passive one. The patchwork of clothing for women in Jiangnan watery region should come from this.

\section{References}

Fujian Museum. (1977). Unearthing bulletin of the tomb of Southern Song Dynasty in the northern suburb of Fuzhou. Cultural Relics, 7, p.4.

$\mathrm{Hu}$, Xiao. (1984). Probe into the culture of folk arts. Publication of Hunan Art, p.433.

Jiangxi Provincial Institute of Cultural Relics and Archaeology. (1990).Unearthing bulletin of Zhou's tomb of Southern Song Dynasty in De'an of Jiangxi. Cultural Relics, 9, p.4.

Taizhou Museum. (1986).Unearthing Bulletin of Fan Xu's tomb of Ming Dynasty in Taizhou of Jiangsu. Cultural Relics, 9, p.3.

Zhang, Jingqiong. (2002).Female folk Clothing in the waterside village Luzhi of "the integration of aesthetic and practical function" based. Journal of Textile Research, 6, PP. 86-88.

Zhang, Jingqiong. (2005).Design and origins of women's head ornaments in Jiangnan watery region. Journal of Textile Research, 5, P. 133.

Zhou, Xibao. (1984). History of ancient Chinese costumes and ornaments. China Theatre Press, p.50.

Zhou, Xibao. (1984). History of ancient Chinese costumes and ornaments.China Theatre Press, P.433.

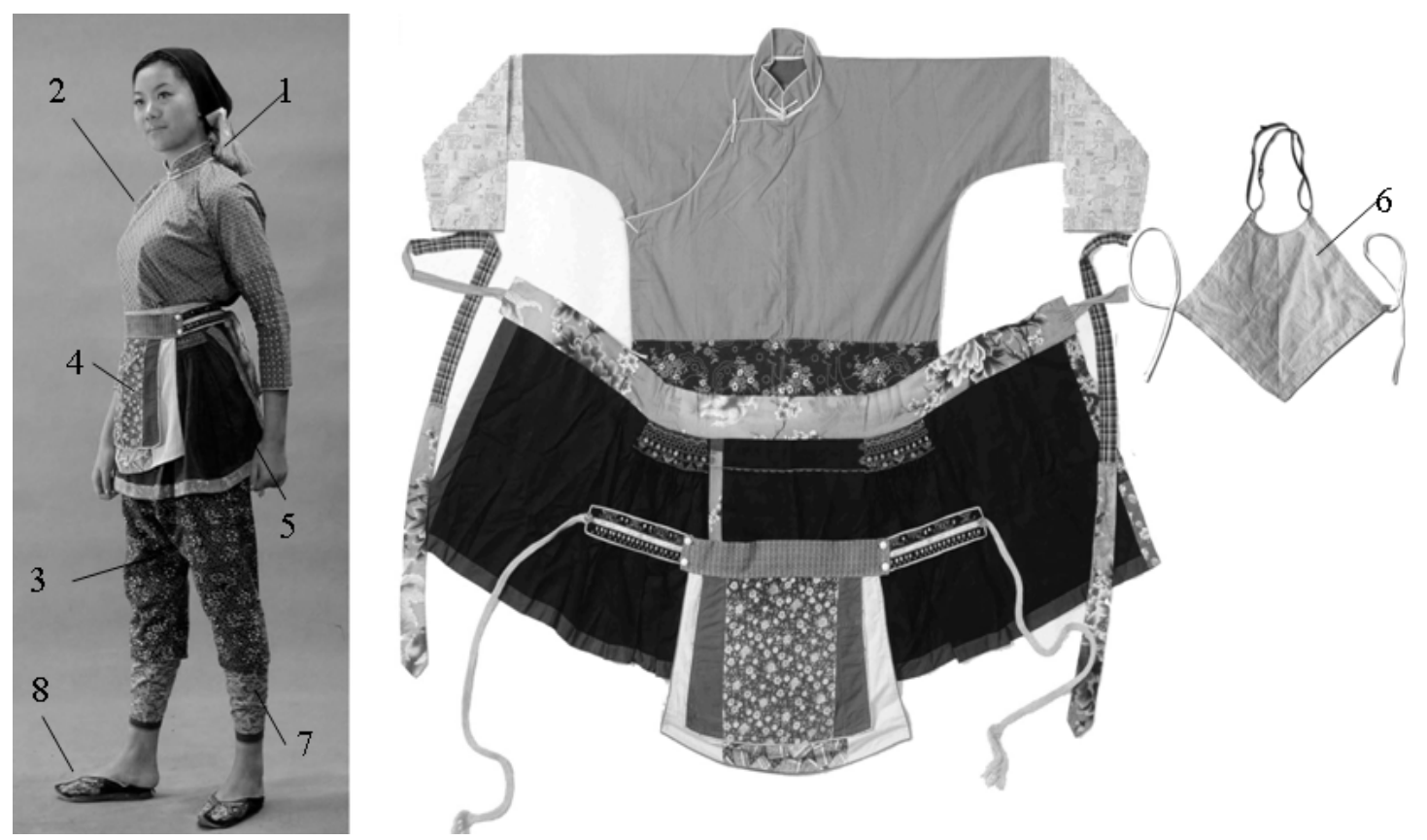

Figure 1. A Complete Set of Clothing for Women in Jiangnan Watery Region 


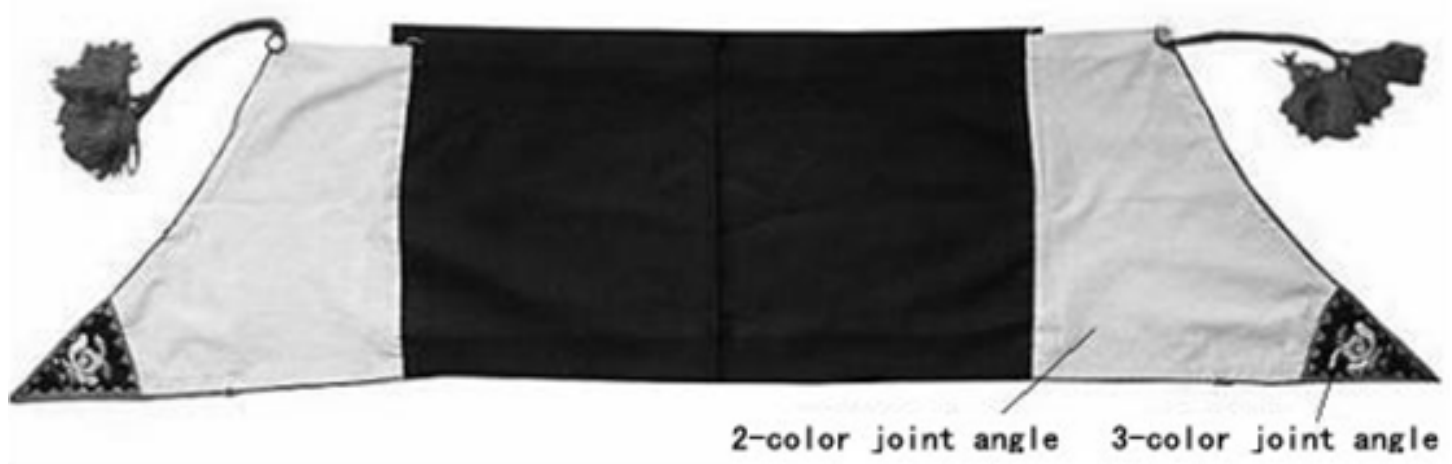

Figure 2. Local Women's Head Scarf

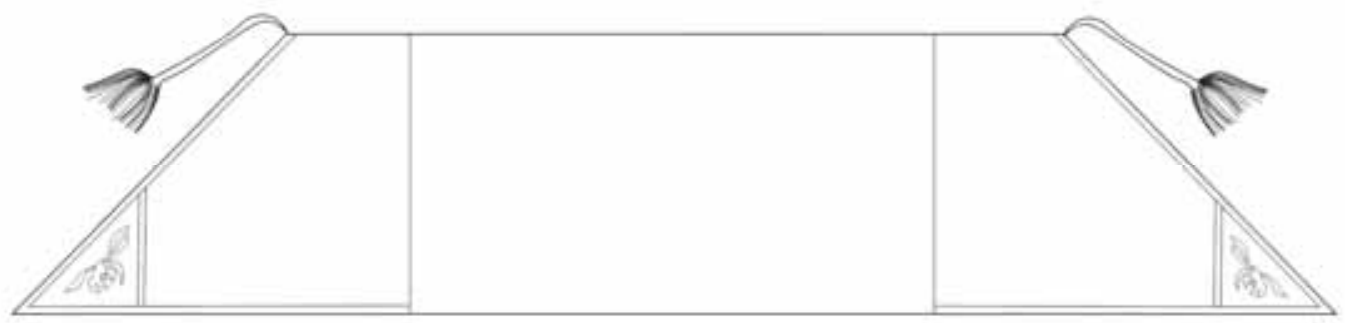

Figure 3. Structure of the Local Head Scarf

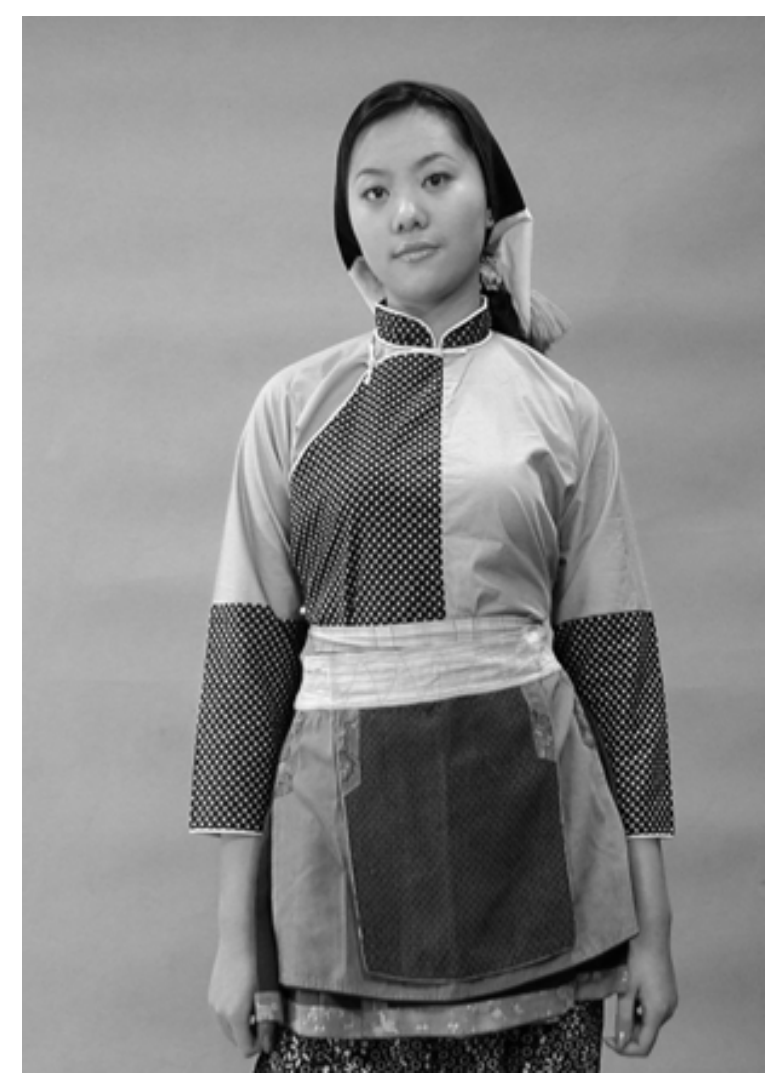

Figure 4. Local Women's Blouse 


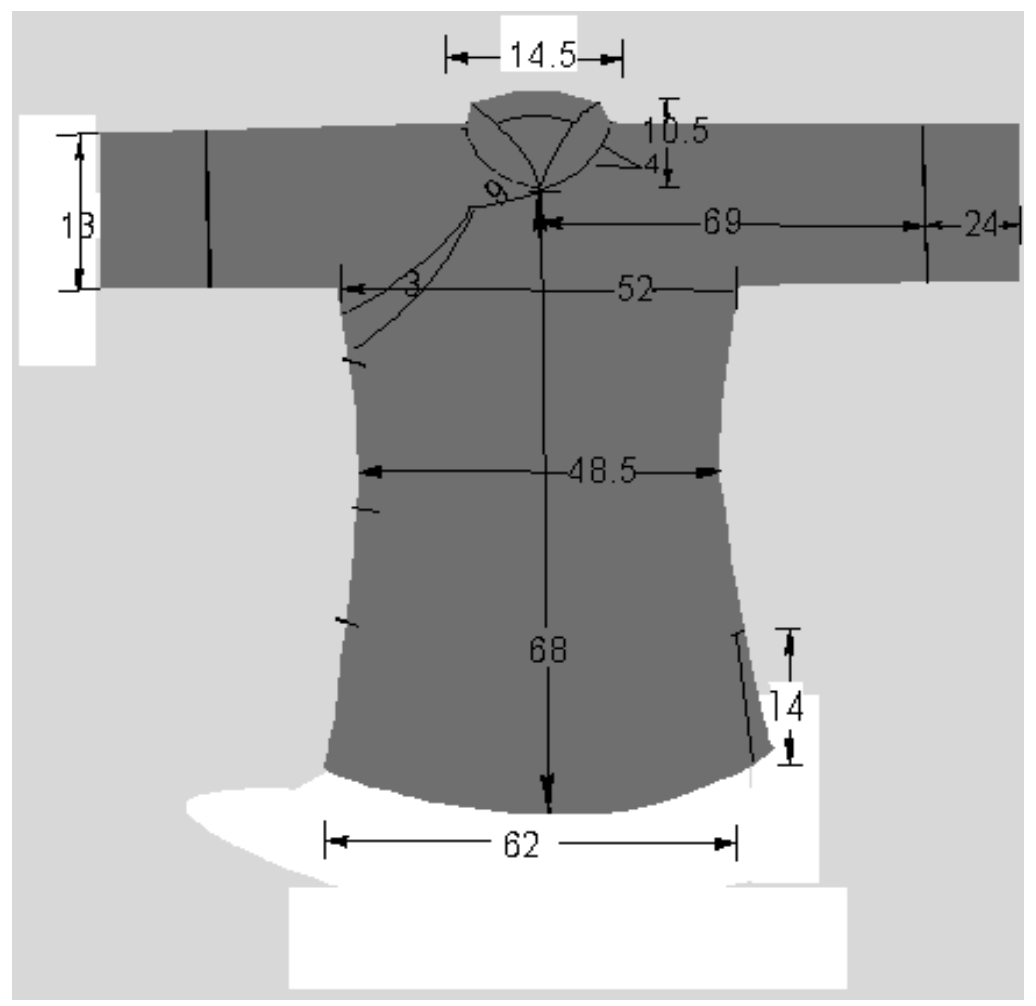

Figure 5. Structure of the Local Blouse

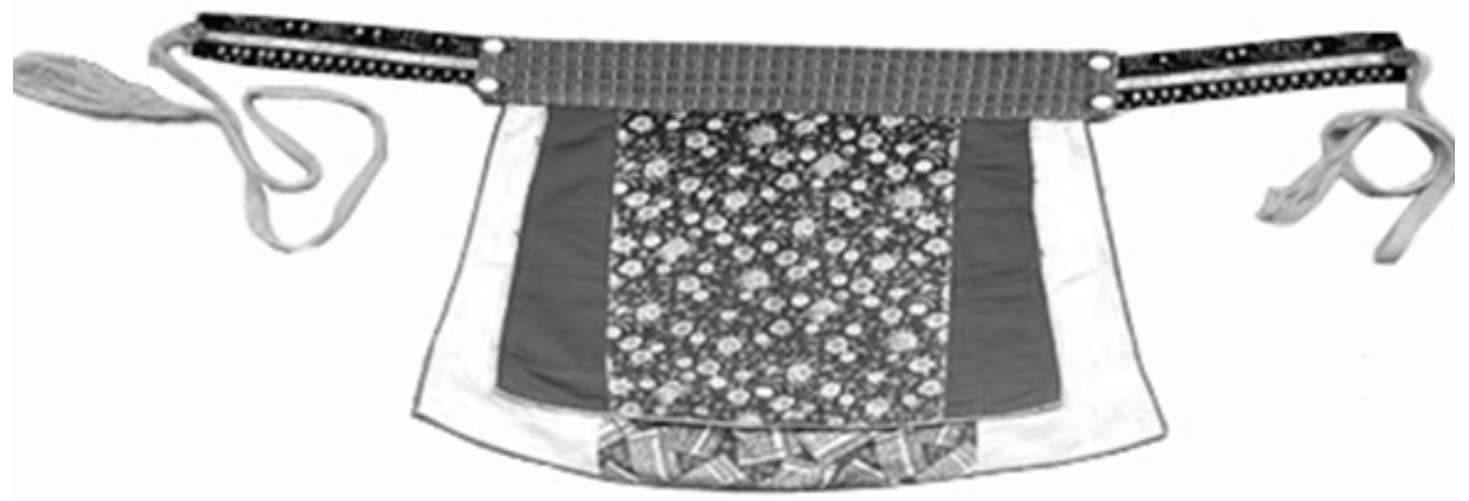

Figure 6. Local Women's Waist-skirt

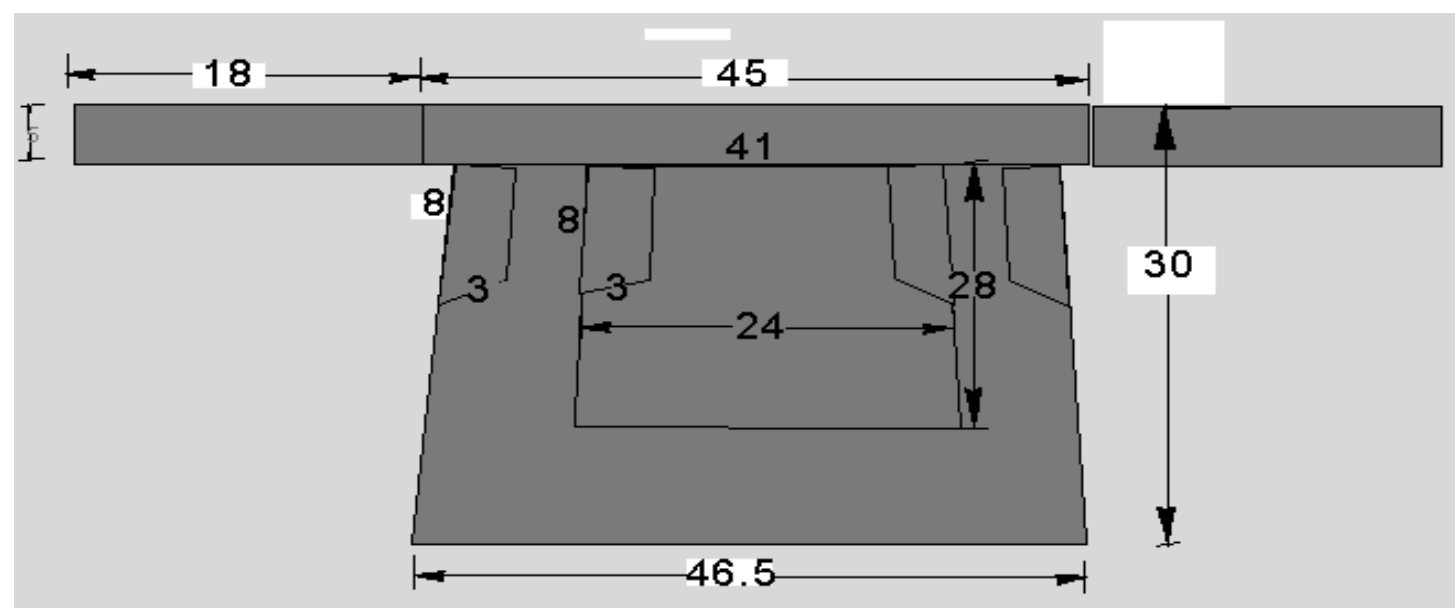

Figure 7. Structure of the Local Waist-skirt 